OPEN ACCESS

Edited by:

Ying Ying Leung

Singapore General

Hospital, Singapore

Reviewed by:

Nelly Raymond Ziade, Saint Joseph University, Lebanon

Miranda Van Lunteren,

Leiden University Medical

Center, Netherlands

*Correspondence:

Jinmei Su

jinmei.su@cstar.org.cn

Xiaofeng Zeng

zengxfpumc@163.com

†These authors have contributed equally to this work

Specialty section:

This article was submitted to Rheumatology,

a section of the journal

Frontiers in Medicine

Received: 23 September 2020 Accepted: 24 November 2020 Published: 23 December 2020

Citation:

Zhang S, Wang Y, Peng L, Su J, Zeng $X, L i M, W u Z, X u J$, Yang $M$, Wu L, Zhao C, Duan X, Li Q, Zhu J and Fan W (2020) Comparison of Clinical Features in HLA-B27 Positive and Negative Patients With Axial Spondyloarthritis: Results From a

Cohort of 4,131 Patients.

Front. Med. 7:609562.

doi: 10.3389/fmed.2020.609562

\section{Comparison of Clinical Features in HLA-B27 Positive and Negative Patients With Axial Spondyloarthritis: Results From a Cohort of 4,131 Patients}

\author{
Shangzhu Zhang ${ }^{1 \dagger}$, Yanhong Wang ${ }^{2 \dagger}$, Linyi Peng ${ }^{1 \dagger}$, Jinmei Su ${ }^{1 *}$, Xiaofeng Zeng ${ }^{1 *}$, \\ Mengtao $\mathrm{Li}^{1}$, Zhenbiao $\mathrm{Wu}^{3}$, Jian $\mathrm{Xu}^{4}{ }^{4}$, Min Yang ${ }^{5}$, Lijun $\mathrm{Wu}^{6}$, Cheng Zhao ${ }^{7}$, \\ Xinwang Duan ${ }^{8}$, Qin $L i^{9}$, Jing Zhu ${ }^{10}$ and Wenqiang Fan ${ }^{11}$
}

\begin{abstract}
${ }^{1}$ Key Laboratory of Rheumatology and Clinical Immunology, Ministry of Education, Ministry of Science and Technology, Department of Rheumatology, National Clinical Research Center for Dermatologic and Immunologic Diseases, Peking Union Medical College, Peking Union Medical College Hospital, Chinese Academy of Medical Sciences, Beijing, China, ${ }^{2}$ Department of Epidemiology and Bio-Statistics (YW), Peking Union Medical College, Institute of Basic Medical Sciences, China Academy of Medical Sciences, Beijing, China, ${ }^{3}$ Department of Rheumatology, Xijing Hospital affiliated to the Fourth Military Medical University, Xi'an, China, ${ }^{4}$ Department of Rheumatology, Kunming Medical University First Affiliated Hospital, Kunming, China, ${ }^{5}$ Department of Rheumatology, Nanfang Hospital Affiliated to Southern Medical University, Guangzhou, China, ${ }^{6}$ Department of Rheumatology, The People's Hospital of Xinjiang Uygur Autonomous Region, Urumchi, China, ${ }^{7}$ Department of Rheumatology, Guangxi Medical University First Affiliated Hospital, Nanning, China, ${ }^{8}$ Department of Rheumatology, Nanchang University Second Affiliated Hospital, Nanchang, China, ${ }^{9}$ Department of Rheumatology, First People's Hospital of Yunnan, Kunming, China, ${ }^{10}$ Department of Rheumatology, Sichuan Provincial People's Hospital, Chengdu, China, ${ }^{11}$ Department of Rheumatology, Xinxiang Central Hospital, Xinxiang, China
\end{abstract}

Objective: The aim of our study was to assess the influence of the HLA-B27 status on axial spondyloarthritis (axSpA) in the largest cohort in China.

Methods: An observational, cross-sectional, and analytic study of axSpA patients from the China axSpA database was performed. Demographic and clinical data were compared in terms of the HLA-B27 status. Univariate and multivariate analyses were performed to identify variables related to HLA-B27 presence.

Results: We enrolled 4,131 patients in this study; of those, 36,95 (89.4\%) were HLA-B27 positive. In the multivariate analysis, male gender $(p<0.001)$, younger age $(p<0.001)$, a disease duration of more than 3 years $(p<0.001)$, a family history of $\operatorname{SpA}(p<0.001)$, uveitis $(p<0.001)$, ASDAS-CRP $(p<0.001)$, and biologic treatment $(p<0.001)$ were the main variables that were independently related to HLA-B27 presence, whereas a diagnosis delay time $>36$ months $(p<0.001)$ and psoriasis $(p<0.001)$ were independently related to HLA-B27 absence.

Conclusion: In Chinese axial SpA patients, presence of HLA-B27 is associated with the male sex, younger age, longer disease duration, greater family aggregation, and higher frequency of uveitis; absence of HLA-B27 is associated with longer diagnosis delay time and higher frequency of psoriasis.

Keywords: HLA-B27, family history, uveitis, psoriasis, axial spondyloarthritis (axSpA) 


\section{INTRODUCTION}

Axial spondyloarthritis ( $\mathrm{SpA}$ ) describes a heterogeneous group of rheumatic diseases characterized by axial skeleton involvement. It can also involve other sites such as peripheral arthritis, enthesitis, dactylitis, and extra-articular manifestations as uveitis, psoriasis, and inflammatory bowel disease (IBD). The term axial spondyloarthritis affects patients with both non-radiographic and radiographic axial spondyloarthritis, which is also termed ankylosing spondylitis (AS) (1).

Investigators have estimated that HLA-B27 in the major histocompatibility complex (MHC) locus contributes to $20.1 \%$ of AS heritability (2). HLA-B27 is found in $74-89 \%$ of patients with either non-radiographic axial spondyloarthritis or ankylosing spondylitis by GESPIC (3) and Herne cohort (4). Moreover, previous studies suggest a relationship between HLAB27 and clinical manifestations. HLA-B27 is also known to be associated with earlier age of axial SpA onset $(3,5)$, increased severity and persistence of MRI-demonstrated inflammation at the sacroiliac joints (SIJ) and lumbar spine in early low back pain (IBP) (6), and anterior uveitis in SpA patients $(5,7)$. However, the clinical characteristics of axial SpA patients related to HLAB27 presence have not been determined in a large cohort of Chinese patients to date. Since previous studies focused mainly on its association with AS, the exact role of HLA-B27 in axial SpA remains unknown in China. Furthermore, the distribution of HLA-B27 population is various. Higher rates of HLA-B27 were found in ax-SpA patients of North America and Western Europe $(80-95 \%)(8)$ than in Japan and Arab countries $(41-84 \%)(9,10)$. Meanwhile, the data for China is limited.

This study aimed to evaluate the HLA-B27 influence on the clinical expression of axial SpA patients. Hence, we reviewed data from the China Spondyloarthritis (ChinSpA) registry database, which includes more than 4,000 axial SpA patients.

\section{MATERIALS AND METHODS}

\section{Patients and Study Design}

The ChinSpA registry is a longitudinal observational study exploring the clinical features, predictors of $\mathrm{SpA}$ activity, and severity of Chinese patients. It is available through a computerized internet database accessible to all participating physician members. The data of this study is from ChinSpA registry. The inclusion criteria of this study were: 1) Patients were required to have a clinical diagnosis of axSpA by a rheumatologist, and fulfilled the classification criteria of Assessment of SpondyloArthritis international Society (ASAS) (11). AxSpA can be subclassified as radiographic axSpA (raxSpA) such as ankylosing spondylitis or non-radiographic axSpA (nr-axSpA) according to the presence or absence of sacroiliac joint damage on X ray (11). The ASAS classification of axSpA relies either on sacroiliitis on imaging plus one SpA feature (imaging arm) or HLA-B27 antigen plus two SpA features (clinical arm), in a patient with chronic low back pain and age at onset of $<45$ years. 2) Patients who were at least 18 years old. 3 ) If the information of the HLA-B27 status were available. It collected baseline data for patients enrolled from departments of Rheumatology in 225 hospitals from 31 provinces in China. Patient recruitment was between August 2018 and May 2020. The study protocol was in accordance with the guidelines of the Helsinki Declaration and was approved by the ethical committee of Peking Union Medical College Hospital and all the other participant hospitals. Written informed consent was obtained from all participants.

All clinical parameters were examined by the rheumatologists in the registry. Patients were interviewed for baseline characteristics which included gender, age at enrollment, age at onset, age at diagnosis, relevant family history, presence of SpA features, extra-articular manifestations, disease activity, medication including use of non-steroidal anti-inflammatory drugs (NSAIDs), disease-modifying antirheumatic drugs (DMARDs), and biologic treatment. Most patients could provide clear information on past clinical manifestation such as age at onset, past peripheral arthritis, and past dactylitis.

Family history was defined as whether the subject had aony first-degree relatives (mother, father, sisters, brothers, and children) or second-degree (maternal and paternal grandparents, aunts, uncles, nieces, and nephews) relatives with history of any of the following: (1) AS; (2) psoriasis; (3) acute uveitis; (4) reactive arthritis; and (5) IBD (11).

A 44-joints count has been proposed to measure peripheral joint involvement, which includes the sternoclavicular joints, acromioclavicular joints, shoulders, elbows, wrists, knees, ankles, MCP, and MTP joints, and PIP joints of the hands. Peripheral arthritis was defined as the presence of swelling in at least one peripheral joint.

The Maastricht Ankylosing Spondylitis Enthesitis Score has been proposed to measure enthesitis involvement (12), which includes costochondral $1 \mathrm{right} / \mathrm{left}$, costochondral $7 \mathrm{right} / \mathrm{left}$, spina iliaca anterior superior right/left, crista iliaca right/left, spina iliaca posterior right/left, processus spinosus L5, achilles tendon, and proximal insertion right/left.

Laboratory tests included C-reactive protein (CRP) and erythrocyte sedimentation rate (ESR) and the HLA-B27 status. Disease activity assessment such as the Bath Ankylosing Spondylitis Disease Activity Index (BASDAI) (13), the Bath Ankylosing Spondylitis Functional Index (BASFI) (14), patient global assessment (PGA), spinal pain, and night back pain were performed. The Ankylosing Spondylitis Disease Activity Score (ASDAS) was calculated with CRP (15).

\section{Statistical Analyses}

All quantitative data were described as mean and standard deviation for the normal distribution or median and interquartile range (IQR) for the non-normal distribution. Additionally, unpaired $T$-tests or Wilcoxon rank-sum tests were performed, respectively, to compare both groups. Frequencies and percentages were used for qualifying data and were compared by chi-square test or Fisher's exact tests.

All statistical analyses were performed using SAS 9.4 (SAS Institute, Cary, NC, USA), and significance was set at $p<0.05$. Statistically significant variables according to univariate analysis were considered in the multiple logistic regression models. Stepwise selection method was adopted for estimating 
TABLE 1 | Baseline demographics, clinical features and treatments in Ax-SpA patients with and without HLA-B27.

\begin{tabular}{|c|c|c|c|}
\hline Variables & With HLA-B27 N=3,695 & Without HLA-B27 N=436 & $p$-value \\
\hline Male, $n(\%)$ & $2,774(75.1)$ & $247(56.7)$ & $<0.001$ \\
\hline Age at enrollment (years), mean $\pm S D$ & $33.22 \pm 10.68$ & $37.35 \pm 13.0$ & $<0.001$ \\
\hline Age of onset (years), mean \pm SD & $26.21 \pm 9.45$ & $31.29 \pm 12.26$ & $<0.001$ \\
\hline Age at diagnosis, years (mean \pm SD) & $29.57 \pm 10.46$ & $34.98 \pm 13.52$ & $<0.001$ \\
\hline Disease duration (years), median (Q1, Q3) & $5(2,10)$ & $3(1,8)$ & $<0.001$ \\
\hline Diagnosis delay time (months), median (Q1, Q3) & $12(1,48)$ & $17.5(2,58.5)$ & 0.034 \\
\hline Family history, $n$ (\%) & $899(24.3)$ & $50(11.5)$ & $<0.001$ \\
\hline \multicolumn{4}{|l|}{ Peripheral manifestations } \\
\hline Current or past peripheral arthritis, $n(\%)$ & $1,321(35.8)$ & $142(32.6)$ & 0.189 \\
\hline Current peripheral arthritis, $n(\%)$ & $999(27.0)$ & $158(36.2)$ & 0.001 \\
\hline Current or past heel pain $n(\%)$ & $832(22.5)$ & $114(26.2)$ & 0.088 \\
\hline Current enthesitis, $n(\%)$ & $2,386(64.6)$ & $297(68.1)$ & 0.146 \\
\hline Current or past dactylitis, $n$ (\%) & $216(6.3)$ & $40(9.7)$ & 0.010 \\
\hline Current hip joint involvement, $n(\%)$ & 1,135 (33.2) & $106(25.7)$ & 0.002 \\
\hline \multicolumn{4}{|l|}{ Extra-articular manifestations } \\
\hline Current or past uveitis, $n(\%)$ & $412(11.2)$ & $22(5.1)$ & $<0.001$ \\
\hline Current or past psoriasis, $n(\%)$ & $27(0.7)$ & $11(2.5)$ & $<0.001$ \\
\hline Current or past inflammatory bowel disease, $n(\%)$ & $55(1.5)$ & $11(2.5)$ & 0.103 \\
\hline BASDAI, mean \pm SD & $3.68 \pm 2.22$ & $3.66 \pm 2.12$ & 0.970 \\
\hline BASFI, median (Q1, Q3) & $2.1(0.6,4.3)$ & $1.9(0.6,3.85)$ & 0.129 \\
\hline CRP, mg/L, median (Q1, Q3) & $10(3.11,24.5)$ & $5(1.42,17.25)$ & $<0.001$ \\
\hline ASDAS-CRP, mean \pm SD & $2.69 \pm 1.23$ & $2.42 \pm 1.21$ & $<0.001$ \\
\hline Good response to NSAIDs, n (\%) & 2,728 (79.7) & $310(75.1)$ & 0.027 \\
\hline NSAIDs & 2,298 (67.2) & 306 (74.1) & 0.004 \\
\hline Biologics & $1,741(50.9)$ & $141(34.1)$ & $<0.001$ \\
\hline DMARDs & 2,073 (60.6) & 268 (64.9) & 0.091 \\
\hline
\end{tabular}

Ax-SpA, Axial spondyloarthritis; BASDAl, Bath Ankylosing Spondylitis Disease Activity Index; BASFI, Bath Ankylosing Spondylitis Functional Index; CRP, C-reactive protein; ESR, erythrocyte sedimentation rate; ASDAS, Ankylosing Spondylitis Disease Activity Score; NSAIDs, non-steroidal anti-inflammatory drugs; HLA-B27, human leukocyte antigen B27.

parameters using $p=0.10$ as a criterion for variable entry and $p=0.05$ as a criterion for variable stay in the model. The associations were represented by odds ratio (OR) and $95 \%$ confidence interval.

\section{RESULTS}

Amon total 4,444 ax-Spa patients, 4,131 (93.0\%) were tested for HLA-B27. Altogether, 4,131 patients were included in this study; of those, 3,695 (89.4\%) with HLA-B27 and 436 without HLA-B27 were analyzed. 609 (14.7\%) patients fulfilled the imaging arm, 207 (5.0\%) fulfilled the clinical arm and 3,315 (80.3\%) fulfilled the both clinical and imaging arms. $73.1 \%$ of all axial patients were male, and the mean disease duration was $6.69 \pm 7.06 \mathrm{y}$. The comparison of characteristics between patients with and without HLA-B27 is shown in Table 1.

The proportion of male is higher in patients with HLA-B27 than in patients without HLA-B27 (75.1 vs. 56.7\%, $p<0.001$ ). Patients with HLA-B27 showed younger age at onset $(p<0.001)$, younger age at diagnosis $(p<0.001)$, longer disease duration $(p<0.001)$, and shorter diagnosis delay time $(p=0.034)$ than patients without HLA-B27. Moreover, patients with HLA-B27 showed a greater prevalence of family history than patients without HLA-B27 (24.3 vs. $11.5 \%, p<0.001$ ) (Table 1).

Compared to the patients without HLA-B27, those with HLAB27 had a significantly higher likelihood of hip joint involvement (33.2 vs. $25.7 \%, p=0.002$ ), but a lower likelihood of peripheral arthritis (27.0 vs. $36.2 \%, p<0.001)$ and dactylitis (6.3 vs. $9.7 \%$, $p=0.01)$ at enrollment. However, no differences were observed between both groups regarding the prevalence of current or past peripheral arthritis. For extra-articular manifestations, patients with HLA-B27 showed a lower prevalence of psoriasis $(0.7$ vs. $2.5 \%, p<0.001)$, a higher prevalence of uveitis (11.2 vs. $5.1 \%, p<0.001)$ than patients without HLA-B27. Furthermore, patients with HLA-B27 expressed a higher disease activity measured by CRP $(p<0.001)$ and ASDAS-CRP $(p<0.001)$. However, this study did not find any statistical differences regarding the BASDAI and BASFI. For treatment, patients with HLA-B27 showed a greater prevalence with biologic treatment (50.9 vs. $34.1 \%, p<0.001$ ), but a lower prevalence with NSAIDs (67.2 vs. $74.1 \%, p=0.004)$ than patients without HLA-B27 at enrollment (Table 1).

In the multivariate analysis, men $(p<0.001)$, younger age $(p<0.001)$, disease duration $>3$ years $(p<0.001)$, family history $(p<0.001)$, uveitis $(p<0.001)$, higher ASDAS-CRP $(p<0.001)$, 
TABLE 2 | Multivariate logistic regression analysis of clinical characteristics of Ax-SpA patients based on the HLA-B27 status.

\begin{tabular}{|c|c|c|c|c|}
\hline \multirow[b]{2}{*}{ Variables* } & \multicolumn{2}{|c|}{ Univariate logistic regression } & \multicolumn{2}{|c|}{ Stepwise logistic regression } \\
\hline & OR $(95 \% \mathrm{Cl})$ & $P$-value & OR $_{\text {adj }}(95 \% \mathrm{Cl})$ & $P$-value \\
\hline Male (ref=male) & $0.43(0.35-0.53)$ & $<0.001$ & $0.58(0.46-0.72)$ & $<0.001$ \\
\hline Age/years & 0.97(0.96-0.98) & $<0.001$ & $0.97(0.96-0.98)$ & $<0.001$ \\
\hline Disease duration $>3$ years $($ ref $="<=3$ years") & $1.45(1.19-1.76)$ & $<0.001$ & $1.84(1.40-2.43)$ & $<0.001$ \\
\hline Diagnosis delay time (<36 vs.<3 months) & $0.79(0.62-1.02)$ & 0.625 & $0.76(0.58-1.00)$ & 0.678 \\
\hline Diagnosis delay time ( $\geq 36$ vs. $<3$ months) & $0.70(0.54-0.90)$ & 0.021 & $0.52(0.38-0.71)$ & $<0.001$ \\
\hline Family history (ref=none) & $2.48(1.83-3.36)$ & $<0.001$ & $2.54(1.84-3.52)$ & $<0.001$ \\
\hline Current peripheral arthritis (ref=none) & $0.65(0.53-0.80)$ & $<0.001$ & $0.61(0.48-0.77)$ & $<0.001$ \\
\hline Dactylitis (ref=none) & $0.63(0.45-0.89)$ & 0.008 & N.A. & N.A. \\
\hline Hip joint involvement (ref=none) & $1.42(1.14-1.78)$ & 0.002 & N.A. & N.A. \\
\hline Psoriasis (ref=none) & $0.28(0.14-0.58)$ & $<0.001$ & $0.18(0.08-0.40)$ & $<0.001$ \\
\hline Uveitis (ref=none) & $2.36(1.52-3.67)$ & $<0.001$ & $2.88(1.81-4.60)$ & $<0.001$ \\
\hline ASDAS-CRP & $1.20(1.10-1.30)$ & $<0.001$ & $1.31(1.19-1.44)$ & $<0.001$ \\
\hline NSAIDs (ref=none) & $0.72(0.57-0.90)$ & 0.005 & N.A. & N.A. \\
\hline Good response to NSAIDs (ref=none) & $1.33(1.05-1.67)$ & 0.016 & N.A. & N.A. \\
\hline Biologics (ref=none) & $2.00(1.61-2.48)$ & $<0.001$ & $1.75(1.40-2.18)$ & $<0.001$ \\
\hline
\end{tabular}

${ }^{*}$ After fully considering collinearity of variables and clinical interpretability, all of these variables were used in stepwise logistic regression, while dactylitis, Hip joint involvement, NSAIDs, and Good response to NSAIDs were not included in final multiple model.

Age and ASDAS-CRP were added as a continuous variable; the statistical method of trisection was used for Diagnosis delay time, and other categorical variables are dichotomous.

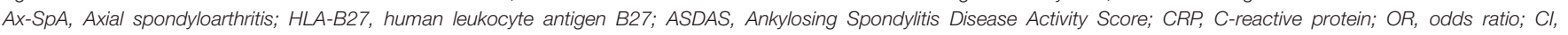
confidence interval.

and on biologic treatment $(p<0.001)$ were the main variables that are independently related to HLA-B27 presence, whereas diagnosis delay time $>36$ months $(p<0.001)$, and psoriasis $(p<0.001)$ were independently related to HLA-B27 absence (Table 2).

\section{DISCUSSION}

Here, we analyzed the clinical characteristics of axial SpA patients according to the HLA-B27 status based on a large dataset of China. We observed a higher proportion of the male sex, younger age, longer disease duration, and family history in axial SpA patients with HLA-B27 than in those without HLA-B27. Moreover, patients with HLA-B27 showed a higher prevalence of uveitis and higher disease activity measured by ASDAS-CRP than in those without HLA-B27. On the other hand, absence of HLAB27 is associated with longer diagnosis delay time and higher frequency of psoriasis.

In all patients with axial $\mathrm{SpA}$ in our study, the prevalence of HLA-B27 presence was $89.4 \%$. A systemic review reported that the prevalence of HLA-B27 in axSpA patients fulfilling any of the contemporary criteria [New York (NY), Amor, ESSG, ASAS] ranged from 26.2 to $91 \%(9,11)$. In addition, in AS patients (radiographic axSpA), the prevalence of HLA-B27 presence was 94.8, 83, 71, and 79\%, in South Korea, Europe, Latin America and Canada, respectively (16-19). The differences possibly due to genetic backgrounds. Moreover, $73.1 \%$ of all patients with axial SpA were male in this study. Similar results were also described in the REGISPONDER, SPARCC, and PSOAS registries $(17,19)$. However, in a Korean study, a higher proportion of the male sex $(88.2 \%)$ was reported in 830 patients with radiographic axial SpA, whereas $58.6 \%$ were male in a French study $(16,20)$. This study found a higher prevalence of male axSpA patients with HLAB27, which is in accordance with that previously published in another Chinese research on radiographic SpA, and also similar in a DESIR cohort conducted in patients representing early forms of the whole spectrum of axial SpA $(20,21)$. However, several other studies did not note any differences $(16,22-24)$, which may be explained by considering geographic and genetic factors.

We observed that the mean age at onset of axial SpA was 5 years earlier in patients with HLA-B27 than in those without HLA-B27 (26 vs. 31 years). The multivariate analysis result showed that younger age was independently related to HLAB27 presence, similarly with previous studies $(5,16,17,20,24$, 25). This study also showed that the diagnosis delay time was significantly longer in patients with HLA-B27 negative compared to patients with HLA-B27 positive, suggesting that HLA-B27 is helpful for the diagnosis of axSpA at early phase. Additionally, our results showed that axial SpA patients with HLA-B27 had greater family aggregation than in those without HLA-B27, which is consistent with previous studies in radiographic axial SpA and in early forms of SpA patients from DESIR (17, 20, $21)$. Furthermore, the finding that $11.5 \%$ of patients without HLA-B27 had family history was in accordance with previous researches, supporting the existence of unknown genetic factors other than HLA-B27 that has a role in the familial aggregation of the disease $(17,20,21)$. 
There was no difference in musculoskeletal manifestations such as peripheral arthritis (current or past), heel pain (current or past), and dactylitis (current or past) between both groups, except a lower prevalence of current peripheral arthritis was found among patients with HLA-B27 at enrollment. In previous studies, the findings regarding the comparison of musculoskeletal manifestations between both groups were inconsistent. In another Chinese research (21), in accordance with ours, the authors found no differences in musculoskeletal manifestations between both groups; whereas in the DESIR cohort (20), the prevalence of present peripheral arthritis was lower in patients with HLA-B27 than in those without HLA-B27. Given that current peripheral arthritis was associated with disease activity, the discrepancy in results on the manifestations may be explained by the different statuses of disease activity upon collection of patient information.

A meta-analysis showed that the prevalence of uveitis in AS patients was associated with disease duration and ranged from $17.4 \%$ to $38.5 \%$ in patients with a mean disease duration of $<10$ and $>20$ years (26). Here, the prevalence of uveitis was $10.5 \%$ in axial $\mathrm{SpA}$ patients with a mean disease duration of 6.9 years. Different geographical areas may explain part of the variation. Furthermore, our result that uveitis was independently related to HLA-B27 presence was in accordance with previous studies $(27,28)$. In contrast, another previous study showed that in patients with uveitis and HLA-B27 presence, the risk of developing $\mathrm{SpA}$ is significantly higher than in uveitis patients without HLA-B27 (29). The prevalence of psoriasis and IBD of this study was 0.9 and $1.6 \%$, respectively, which were lower than those reported in previous studies (26). The discrepancy of prevalence may be due to differences in disease duration and geographical area. Moreover, we did not observe any differences in IBD presence between both groups. Previous study reported a lower prevalence of IBD among patients with HLA-B27 (17). However, no clear evidence has been found to prove that the risk of developing IBD or subclinical IBD is higher in axSpA patients with HLA-B27 (8). Additionally, this study showed that the prevalence of psoriasis was lower in patients with HLA-B27 than in those without HLA-B27, which was consistent with previous studies $(17,20)$. Psoriasis being negatively associated with HLA$\mathrm{B} 27$ presence may be due to the selection bias of patients without HLA-B27 according to the Amor criteria as they require more extra-articular features in order to be diagnosed with SpA (20).

Regarding disease severity, the results reported were inconsistent. Some studies reported similar BASDAI and BASFI scores in patients with and without HLA-B27 (16, 24, 25), whereas other studies showed worse BASDAI and BASFI scores in patients without HLA-B27 (17). Here, we observed no differences in BASDAI and BASFI scores in both groups but a higher level of ASDAS-CRP in patients with HLA-B27. However, a positive correlation between presence of HLA-B27 and high ASDAS-CRP was not previously confirmed (20, 30, 31). The results regarding disease severity depend on patient status and treatment upon data collection. To confirm whether HLA-B27 influences the disease severity, longitudinal studies using average scores of the BASDAI and BASFI should be conducted in the future.
For treatment, no difference was observed between both groups regarding satisfactory response to NSAIDs in multivariate analysis at enrollment. However, no similar research has been found in the past. Previous studies showed that patients with HLA-B27 show a better clinical response to Tumor necrosis factor inhibitor (TNF) inhibitors (32-34). This was a crosssectional study and did not evaluate the therapeutic effect. Furthermore, the results showed that patients with HLA-B27 used more biological agents than did those without HLA-B27 at enrollment, which may be because of higher disease activity of those with HLA-B27 as discussed earlier.

This study has some limitations that should be noted. First, this is a cross-sectional study, and status of disease activity only represented the moment assessing the disease activity. Analyzing whether the HLA-B27 status influences the disease severity using a mean of BASDAI and BASFI scores in a longitudinal cohort may yield more accurate results. We did not analyze the treatment response using TNF alpha inhibitors, which also need a longitudinal cohort. Second, the mean disease duration of patients in this study was 6.9 years. Since some clinical manifestations such as uveitis were associated with disease duration, the results of patients with longer disease duration must be interpreted with caution. Third, only patients with available HLA-B27 were included in the analysis. The patients without any available HLA-B27 might have different characteristics, which may influence the results. Lastly, we did not analyze the influence of HLA-B27 on structural damage.

\section{CONCLUSION}

In summary, this is an extensive study assessing the influence of the HLA-B27 status on axial SpA in the largest Chinese cohort. Our study confirms HLA-B27 is associated, in axial SpA patients, with the male sex, younger age, longer disease duration, greater family aggregation, and higher frequency of uveitis. However, we don't support an association between the HLA-B27 status and any musculoskeletal manifestations. Meanwhile, absence of HLA-B27 is associated with longer diagnosis delay time and higher frequency of psoriasis.

\section{DATA AVAILABILITY STATEMENT}

The raw data supporting the conclusions of this article will be made available by the authors, without undue reservation.

\section{ETHICS STATEMENT}

The studies involving human participants were reviewed and approved by ethical committee of Peking Union Medical College Hospital and all the other participant hospitals. The patients/participants provided their written informed consent to participate in this study. 


\section{AUTHOR CONTRIBUTIONS}

SZ wrote the paper. JS and $\mathrm{XZ}$ were involved in the protocol development, study design, supervision of the data collection, critical revision, reviewing of the report, and quality checking. ML and LP reviewed the paper. YW performed the statistical analysis. ZW, JX, MY, LW, CZ, XD, QL, and WF contributed to the data collection. All authors read and approved the final manuscript.

\section{REFERENCES}

1. Sieper J, Braun J, Dougados M, Baeten D. Axial spondyloarthritis. Nat Rev Dis Primers. (2015) 1:15013. doi: 10.1038/nrdp.2015.13

2. International Genetics of Ankylosing Spondylitis C, Cortes A, Hadler J, Pointon JP, Robinson PC, Karaderi T, et al. Identification of multiple risk variants for ankylosing spondylitis through high-density genotyping of immune-related loci. Nat Genet. (2013) 45:730-8. doi: 10.1038/ng.2667

3. Rudwaleit M, Haibel H, Baraliakos X, Listing J, Marker-Hermann E, Zeidler $\mathrm{H}$, et al. The early disease stage in axial spondylarthritis: results from the German Spondyloarthritis Inception Cohort. Arthritis Rheum. (2009) 60:71727. doi: 10.1002/art.24483

4. Kiltz U, Baraliakos X, Karakostas P, Igelmann M, Kalthoff L, Klink C, et al. Do patients with non-radiographic axial spondylarthritis differ from patients with ankylosing spondylitis? Arthritis Care Res. (2012) 64:141522. doi: 10.1002/acr.21688

5. Feldtkeller E, Khan MA, van der Heijde D, van der Linden S, Braun J. Age at disease onset and diagnosis delay in HLA-B27 negative vs. positive patients with ankylosing spondylitis. Rheumatol Int. (2003) 23:616. doi: 10.1007/s00296-002-0237-4

6. Marzo-Ortega H, McGonagle D, O'Connor P, Hensor EM, Bennett AN, Green $\mathrm{MJ}$, et al. Baseline and 1-year magnetic resonance imaging of the sacroiliac joint and lumbar spine in very early inflammatory back pain. Relationship between symptoms, HLA-B27 and disease extent and persistence. Ann Rheum Dis. (2009) 68:1721-7. doi: 10.1136/ard.2008.097931

7. Sampaio-Barros PD, Conde RA, Bonfiglioli R, Bertolo MB, Samara AM. Characterization and outcome of uveitis in 350 patients with spondyloarthropathies. Rheumatol Int. (2006) 26:1143-6. doi: 10.1007/s00296-006-0203-7

8. Fahed H, Mauro D, Ciccia F, Ziade NR. What does human leukocyte antigen B27 have to do with spondyloarthritis? Rheum Dis Clin North Am. (2020) 46:225-39. doi: 10.1016/j.rdc.2020.01.002

9. Ziade NR. HLA B27 antigen in Middle Eastern and Arab countries: systematic review of the strength of association with axial spondyloarthritis and methodological gaps. BMC Musculoskelet Disord. (2017) 18:280. doi: 10.1186/s12891-017-1639-5

10. Ziade N, Abi Karam G, Merheb G, Mallak I, Irani L, Alam E, et al. HLAB27 prevalence in axial spondyloarthritis patients and in blood donors in a Lebanese population: results from a nationwide study. Int J Rheum Dis. (2019) 22:708-14. doi: 10.1111/1756-185X.13487

11. Rudwaleit M, van der Heijde D, Landewe R, Listing J, Akkoc N, Brandt J, et al. The development of Assessment of SpondyloArthritis international Society classification criteria for axial spondyloarthritis (part II): validation and final selection. Ann Rheum Dis. (2009) 68:777-83. doi: 10.1136/ard.2009.108233

12. Heuft-Dorenbosch L, Spoorenberg A, van Tubergen A, Landewe R, van ver Tempel $H$, Mielants $H$, et al. Assessment of enthesitis in ankylosing spondylitis. Ann Rheum Dis. (2003) 62:127-32. doi: 10.1136/ard. 62.2 .127

13. Garrett S, Jenkinson T, Kennedy LG, Whitelock H, Gaisford P, Calin A. A new approach to defining disease status in ankylosing spondylitis: the bath ankylosing spondylitis disease activity index. J Rheumatol. (1994) 21:2286-91.

14. Calin A, Jones SD, Garrett SL, Kennedy LG. Bath ankylosing spondylitis functional index. $\mathrm{Br} J$ Rheumatol. (1995) 34:7934. doi: $10.1093 /$ rheumatology/34.8.793

\section{FUNDING}

This work was supported by grants from CAMS Innovation Fund for Medical Sciences (2019-I2M-2-008).

\section{ACKNOWLEDGMENTS}

We thank all patients contributing to this study, and all the 225 centers contributing ChinAS registry database.

15. Lukas C, Landewe R, Sieper J, Dougados M, Davis J, Braun J, et al. Development of an ASAS-endorsed disease activity score (ASDAS) in patients with ankylosing spondylitis. Ann Rheum Dis. (2009) 68:1824. doi: 10.1136/ard.2008.094870

16. Kim TJ, Kim TH. Clinical spectrum of ankylosing spondylitis in Korea. Joint Bone Spine. (2010) 77:235-40. doi: 10.1016/j.jbspin.2009.11.015

17. Arevalo M, Gratacos Masmitja J, Moreno M, Calvet J, Orellana C, Ruiz D, et al. Influence of HLA-B27 on the ankylosing spondylitis phenotype: results from the REGISPONSER database. Arthritis Res Ther. (2018) 20:221. doi: 10.1186/s13075-018-1724-7

18. Benegas M, Munoz-Gomariz E, Font P, Burgos-Vargas R, Chaves J, Palleiro $\mathrm{D}$, et al. Comparison of the clinical expression of patients with ankylosing spondylitis from Europe and Latin America. J Rheumatol. (2012) 39:231520. doi: 10.3899/jrheum.110687

19. Gladman DD, Rahman P, Cook RJ, Shen H, Zummer M, Thomson G, et al. The Spondyloarthritis Research Consortium of Canada registry for spondyloarthritis. J Rheumatol. (2011) 38:1343-8. doi: 10.3899/jrheum.101102

20. Chung HY, Machado P, van der Heijde D, D'Agostino MA, Dougados M. HLA-B27 positive patients differ from HLA-B27 negative patients in clinical presentation and imaging: results from the DESIR cohort of patients with recent onset axial spondyloarthritis. Ann Rheum Dis. (2011) 70:19306. doi: 10.1136/ard.2011.152975

21. Yang $M, X u$ M, Pan X, Hu Z, Li Q, Wei Y, et al. Epidemiological comparison of clinical manifestations according to HLA-B*27 carrier status of Chinese ankylosing spondylitis patients. Tissue Antigens. (2013) 82:33843. doi: $10.1111 / \tan .12186$

22. Sampaio-Barros PD, Bertolo MB, Kraemer MH, Neto JF, Samara AM. Primary ankylosing spondylitis: patterns of disease in a Brazilian population of 147 patients. J Rheumatol. (2001) 28:560-5.

23. Nazarinia MA, Ghaffarpasand F, Heiran HR, Habibagahi Z. Pattern of ankylosing spondylitis in an Iranian population of 98 patients. Mod Rheumatol. (2009) 19:309-15. doi: 10.3109/s10165-009-0153-5

24. Kim TJ, Na KS, Lee HJ, Lee B, Kim TH. HLA-B27 homozygosity has no influence on clinical manifestations and functional disability in ankylosing spondylitis. Clin Exp Rheumatol. (2009) 27:574-9.

25. Jaakkola E, Herzberg I, Laiho K, Barnardo MC, Pointon JJ, Kauppi M, et al. Finnish HLA studies confirm the increased risk conferred by HLA-B27 homozygosity in ankylosing spondylitis. Ann Rheum Dis. (2006) 65:77580. doi: 10.1136/ard.2005.041103

26. Stolwijk C, van Tubergen A, Castillo-Ortiz JD, Boonen A. Prevalence of extra-articular manifestations in patients with ankylosing spondylitis: a systematic review and meta-analysis. Ann Rheum Dis. (2015) 74:6573. doi: 10.1136/annrheumdis-2013-203582

27. D'Ambrosio EM, La Cava M, Tortorella P, Gharbiya M, Campanella M, Iannetti L. Clinical features and complications of the HLA-B27-associated acute anterior uveitis: a metanalysis. Semin Ophthalmol. (2017) 32:689701. doi: 10.3109/08820538.2016.1170158

28. Valls Pascual E, Fontanilla Ortega P, Vicens Bernabeu E, Martinez-Costa L, Blanco Alonso R. Clinical characteristics, treatment and ocular complications of HLA-B27-related anterior uveitis and HLA-B27-non related anterior uveitis. Reumatol Clin. (2016) 12:244-7. doi: 10.1016/j.reumae.2015.11.014

29. Accorinti M, Iannetti L, Liverani M, Caggiano C, Gilardi M. Clinical features and prognosis of HLA B27-associated acute anterior uveitis in 
an Italian patient population. Ocul Immunol Inflamm. (2010) 18:916. doi: 10.3109/09273941003597268

30. Sheehan NJ. HLA-B27: what's new? Rheumatology. (2010) 49:621-31. doi: 10.1093/rheumatology/kep450

31. Ruiz DG, Azevedo MN, Lupi O. HLA-B27 frequency in a group of patients with psoriatic arthritis. An Bras Dermatol. (2012) 87:84750. doi: 10.1590/S0365-05962012000600004

32. Rudwaleit M, Claudepierre P, Wordsworth P, Cortina EL, Sieper J, Kron $\mathrm{M}$, et al. Effectiveness, safety, and predictors of good clinical response in 1250 patients treated with adalimumab for active ankylosing spondylitis. $J$ Rheumatol. (2009) 36:801-8. doi: 10.3899/jrheum.081048

33. Vastesaeger N, van der Heijde D, Inman RD, Wang Y, Deodhar A, Hsu B, et al. Predicting the outcome of ankylosing spondylitis therapy. Ann Rheum Dis. (2011) 70:973-81. doi: 10.1136/ard.2010.147744

34. Baraliakos X, Koenig AS, Jones H, Szumski A, Collier D, Bananis E. Predictors of clinical remission under anti-tumor necrosis factor treatment in patients with ankylosing spondylitis: pooled analysis from large randomized clinical trials. J Rheumatol. (2015) 42:1418-26. doi: 10.3899/jrheum.14 1278

Conflict of Interest: The authors declare that the research was conducted in the absence of any commercial or financial relationships that could be construed as a potential conflict of interest.

Copyright (c) 2020 Zhang, Wang, Peng, Su, Zeng, Li, Wu, Xu, Yang, Wu, Zhao, Duan, Li, Zhu and Fan. This is an open-access article distributed under the terms of the Creative Commons Attribution License (CC BY). The use, distribution or reproduction in other forums is permitted, provided the original author(s) and the copyright owner(s) are credited and that the original publication in this journal is cited, in accordance with accepted academic practice. No use distribution or reproduction is permitted which does not comply with these terms. 